\title{
ROLE OF COLOR DOPPLER ULTRASONOGRAPHY VERSUS TRIPHASIC CT WITH PORTOGRAPHY IN PORTAL VEIN THROMBOSIS
}

\author{
Eman A. Abdelsalam; Maha M. Abdelraof; Mohamed S. Hassan \\ and Khaled A.M. Ali
}

Radiology department, Ain Shams University, Cairo, Egypt

Corresponding Author:

Eman Abdeladl Mohamed

Email:

imanmohamed2005@gmail.co $\underline{\mathrm{m}}$.

Mob. +201090810640

Received: $29 / 8 / 2020$

Accepted: 15/9/2020

Online ISSN: 2735-3540

\begin{abstract}
:
Back ground: Although portal vein thrombosis is a rare event among general population, its prevalence in cirrhotic patients about $28 \%$ (1). Generally liver cirrhosis is a widespread disease, the differentiation between bland and malignant thrombus is very essential as neoplastic portal vein thrombosis gravely affects the prognosis and the subsequent treatment options (1). Doppler ultrasonography usually the first noninvasive modality used to diagnose portal vein thrombosis, using color and spectral data analysis. Triphasic CT with portography is very helpful to differentiate bland from malignant thrombus and enable characterization of malignant portal vein thrombosis using maximum intensity projection (MIP) reconstruction allow better analysis of the thrombus (2).
\end{abstract}

Aim of the work: To show the role of color Doppler ultrasonography (US) and Triphasic CT in diagnosis of portal vein thrombosis (PVT) and differentiation between bland and tumoral thrombus.

Patient and Methods: This study included 32 cases presented with portal vein thrombosis clinically and radiologically.

Results: Of the 32 subjects, 21 had malignant PVT and 11 patients had benign PVT. Direct invasion of portal vein from adjacent focal lesion had the highest sensitivity $95.2 \%$ was achieved, with a specificity of $100 \%$. The Positive predictive value (PPV) was $100 \%$, while the negative predictive value (NPV) was $91.7 \%$. Early arterial enhancement and rapid washout of contrast showed $76.2 \%$ sensitivity and $100 \%$ specificity, PPV $100 \%$ and NPV $68.8 \%$. Pulsatile flow within the thrombus depicted $38.1 \%$ sensitivity, $100 \%$ specificity with PPV $100 \%$ and NPV $45.8 \%$.

Conclusion: Triphasic CT and color Doppler US have to be considered a useful tools in evaluating portal vein thrombosis provides helpful information for the detection and characterization of malignant and benign portal vein thrombi.

Keywords: Portal vein thrombosis (PVT); color Doppler US; Triphasic Computed tomography (CT); malignant and benign portal vein thrombosis.

\section{INTRODUCTION:}

Portal vein thrombosis (PVT) indicates partial or complete occlusion of portal vein or its branches by a thrombus within its lumen $^{(1)}$. It occurs either with liver cirrhosis or with hepatic malignancy ${ }^{(2)}$. The differentiation between tumoral and bland PVT is 
crucial to determine tumor staging and subsequent treatment options ${ }^{(3)}$. Although the reference standard for characterization of PVT is histopathological examination, in clinical practice various diagnostic imaging modalities including Doppler US and Triphasic CT, together with clinical and laboratory findings is often relied upon for thrombus discrimination ${ }^{(1)}$. Usually Doppler US is the initial available non invasive technique used to assess portal vein ${ }^{(4)}$. Diagnosis of malignant PVT was evaluated by several studies, by detection of pulsatile flow within intraluminal thrombus ${ }^{5)}$. Using Triphasic CT with early and late arterial, portal venous and delayed phases with fixed time delays is quick, easy and non invasive useful imaging modality in thrombus characterization, direct invasion of portal vein by adjacent focal lesion, detection of arterial enhancement and rapid washout of contrast with expansion of portal vein with the thrombus ${ }^{(6)}$.

\section{PATIENTS AND METHODS:}

This study was approved by the ethics committee of our institution. This study carried out on 32 patients done at private centers and Ain Shams Hospital radiology department between December 2017 and June 2020.

\section{Study population:}

Patients subjected to the stud according to the following criteria:

\section{Inclusion criteria:}

Both genders and all ages were included. Patients with chronic hepatic liver disease. Patients with non cirrhotic liver presenting with PVT. Pathologically or radiologically proven HCC. Patient with visible portal vein thrombosis in US.

\section{Exclusion criteria:}

Patients with impaired renal function or (serum creatinine level more than 1.5 or
GFR less than $30 \mathrm{~mL} / \mathrm{min} / 1.73 \mathrm{~m} 2$ ).

\section{Patient preparation:}

Fasting 4-6 h prior to Doppler and CT examination to optimize imaging and avoid complications with IV contrast injection.

\section{Imaging techniques:}

\section{1) Color Doppler ultrasonography:}

Real time ultrasound: Abdominal ultrasound was done with (General Electric medical system, Toshiba) ultrasound machine using $3.5 \mathrm{MHz}$ transducer for examination of the liver as regards its size, echo texture, contour, hepatic focal lesions, portal vein patency, the presence of intra or extra hepatic biliary radicle dilatation, porta hepatis or abdominal lymph nodes and ascites. Spleen, splenic hilum and pancreas also evaluated.

Color Doppler ultrasonography of portal vein: Color Doppler US was performed using $3.5 \mathrm{MHz}$ matched imaging and Doppler frequency transducer. The protocol consists of obtaining gray scale image of PV thrombus and performing color, power and duplex Doppler US for PV thrombus, PV tributaries and branches to determine thrombus extension and adjacent hepatic artery. Thrombi in left portal vein were evaluated using an anterior sub-xiphoid approach, and right portal vein thrombi evaluated between the mid-clavicular line and the posterior axillary line using intercostal or subcostal approach. The flow was categorized by spectral configuration as pulsatile or continuous. Pulsatile flow was called subjectively when a waveform had a marked difference between peak systolic and end diastolic velocities.

\section{2) CT Imaging Technique:}

Triphasic CT of the abdomen and pelvis was performed using MDCT scanner 16 detectors (GE, Philips Philips Health care) scanner. 
Double arterial phase scanning was done via a power injector, $150 \mathrm{ml}$ of omnipaque $300 \mathrm{mg} / \mathrm{ml}$ (iohexol $300 \mathrm{mg} \mathrm{I} /$ $\mathrm{ml}$; Nycomed, Princeton, NJ) was injected at a rate of $4 \mathrm{ml} / \mathrm{s}$. Early and late arterial phase image acquisition was performed during a single breathe hold using respectively, 20 and $35 \mathrm{~s}$ scanning delays from initiation of contrast material injection, we use single breathe hold to minimize the effect of variation on lesion conspicuity and detection. Portal venous phase was performed using scanning delay of $60 \mathrm{~s}$ and an image thickness of $5 \mathrm{~mm}$ and delayed phase was taken 6-10 minutes after bolus tracking. The entire liver was scanned in craniocaudal direction using detector collimation of 16-1.5 mm, with a table speed/ rotation of $15 \mathrm{~mm} / 0.8 \mathrm{~s}$, and an image thickness of $5 \mathrm{~mm}$. Reconstruction section thickness $0.625 \mathrm{~mm}$, reconstruction interval $0.625 \mathrm{~mm}$, field of view $350 \mathrm{~mm}$, matrix 512-512, $2.0 \mathrm{~mm}$ thin slice thickness of portal venous phase image and $1.0 \mathrm{~mm}$ slice interval, window width $350 \mathrm{HU}$ and window level 50HU. The scanning parameters used were $120 \mathrm{KV}$ and 150-250 $\mathrm{mAs}$ and the pitch value were 0.813. The scan data was transferred to the postprocessing extended Brilliance Workspace (EBW) work station and then the values were measured.

\section{Imaging evaluation}

PVT is diagnosed when an anechoic or hyperechoic structure seen within portal vein lumen with absence of flow on color Doppler US. Partial or complete filling defect within PV on enhanced CT images. We considered portal vein thrombosis as a malignant thrombus when at least two of the following criteria is present:

A. Portal vein thrombus in US with detection of arterial wave form within the thrombus on both color and spectral analysis.
B. PVT seen in continuity with direct invasion by hepatic focal lesion in US or CT.

C. Presence of early arterial enhancement with rapid washout within the thrombus in CT. Otherwise, the thrombus is considered bland thrombus.

\section{Statistical analysis:}

Statistical analysis was performed using Med Calc statistical software for Windows (Med Calc Software, Mariakerke, Belgium). Data for continuous variables were expressed as either median, interquartile range and range or mean \pm standard deviation and as both number and percentage for categorical data. Mann - Whitney and the independent samples $\mathrm{T}$ - test were used to evaluate the differences in quantitative variables when the data was not normally and normally distributed respectively. Categorical data were evaluated using the Chi - squared test. Receiver operator characteristic (ROC) curve analysis was performed to determine the diagnostic accuracy of the various variables in distinguishing the different groups. The diagnostic accuracy of all variables was evaluated in terms of sensitivity, specificity, positive predictive value (PPV), negative predictive value (NPV), and area under the ROC curve (AUC). For all tests all $\mathrm{P}$ values were twotailed and a $\mathrm{P}$-value $<0.05$ was considered significant.

\section{RESULTS:}

32 patients (25 males and 7 females) were included in this study. Histopathological analysis revealed that Eleven (34.4\%) and $21(65.6 \%)$ patients had benign and malignant portal vein thrombosis respectively. Direct invasion of portal vein from adjacent focal lesion had the highest sensitivity $95.2 \%$ was achieved, with a specificity of $100 \%$. The Positive predictive value (PPV) was $100 \%$, while the negative predictive value (NPV) was $91.7 \%$. Early 
arterial enhancement and rapid washout of contrast showed $76.2 \%$ sensitivity and 100 $\%$ specificity, PPV $100 \%$ and NPV $68.8 \%$.
Pulsatile flow within the thrombus depicted $38.1 \%$ sensitivity, $100 \%$ specificity with PPV $100 \%$ and NPV $45.8 \%$.

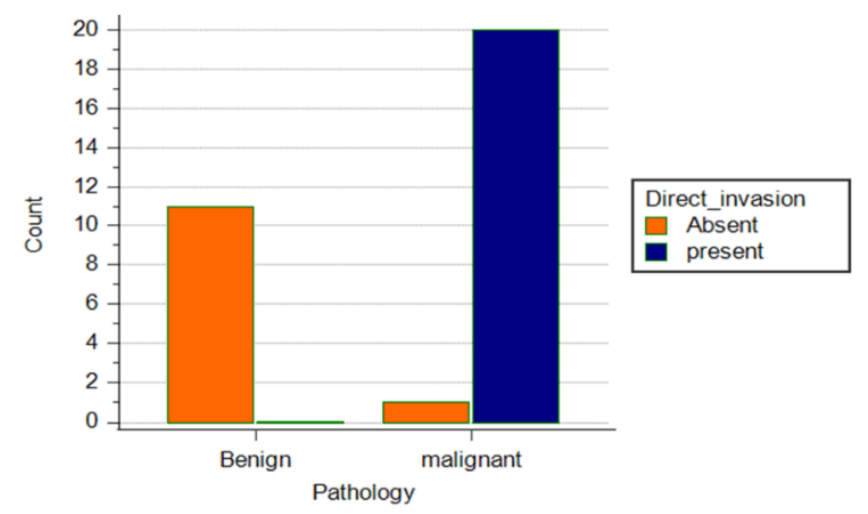

Fig.1: Clustered column chart showing the incidence of direct invasion of the PV by an adjacent mass in benign and malignant PVT.

The diagnostic performance of all parameters in differentiation of malignant PVT from benign PVT as determined by the number of true positive (TP), true negative (TN), false positive (FP), and false negative (FN) cases as well as the calculated sensitivities, specificities, positive predictive values (PPV), negative predictive values (NPV), accuracies, area under curves (AUC) and their associated P values of all parameters.

\begin{tabular}{|l|c|c|c|c|c|c|c|c|c|c|c|}
\hline \multicolumn{1}{|c|}{ Parameter } & TP & TN & FP & FN & Sensitivity & Specificity & PPV & NPV & Accuracy & AUC & $\begin{array}{c}\text { P- } \\
\text { value }\end{array}$ \\
\hline Pulsatile flow & 8 & 11 & 0 & 13 & $38.1 \%$ & $100 \%$ & $100 \%$ & $45.8 \%$ & $59.4 \%$ & 0.69 & $<0.001$ \\
\hline Neovascularity & 7 & 11 & 0 & 14 & $33.3 \%$ & $100 \%$ & $100 \%$ & $44.0 \%$ & $56.3 \%$ & 0.67 & 0.002 \\
\hline $\begin{array}{l}\text { Arterial enh. } \\
\text { \& washout }\end{array}$ & 16 & 11 & 0 & 5 & $76.2 \%$ & $100 \%$ & $100 \%$ & $68.8 \%$ & $84.4 \%$ & 0.88 & $<0.001$ \\
\hline $\begin{array}{l}\text { Direct } \\
\text { invasion }\end{array}$ & 20 & 11 & 0 & 1 & $95.2 \%$ & $100 \%$ & $100 \%$ & $91.7 \%$ & $96.9 \%$ & 0.98 & $<0.001$ \\
\hline $\begin{array}{l}\text { Diameter } \\
>18 \mathrm{~mm}^{\mathrm{a}}\end{array}$ & 13 & 10 & 1 & 8 & $61.9 \%$ & $90.9 \%$ & $92.9 \%$ & $55.6 \%$ & $71.9 \%$ & 0.76 & 0.01 \\
\hline
\end{tabular}

Variable diagnostic criteria were evaluated for differentiation of malignant PVT from benign PVT, the area under their ROC curves were compared. Direct invasion of the PV by an adjacent hepatic mass had the largest AUC followed by arterial enhancement and early washout, a thrombus diameter $>18 \mathrm{~mm}$, intra-thrombus pulsatile flow and finally thrombus neovascularity (FIG). Though the AUC of direct invasion was greater than that of arterial enhancement and washout, no significant difference was between their respective AUCs indicating that the diagnostic performance of these two parameters did not differ significantly. However the AUCs of direct invasion and arterial enhancement were significantly greater than the AUCs of intra-thrombus pulsatile flow and neovascularity indicating that direct invasion and arterial enhancement were better than pulsatile flow and neovascularity in differentiation of malignant and benign PVT. No significant differences were seen between the AUCs of pulsatile flow, neovascularity and diameter or between the AUCs of arterial enhancement and diameter, yet the AUC of direct invasion was significantly greater than the AUC of diameter. Details of the pairwise comparisons of all ROC curves and their statistical significance are listed in the table. 


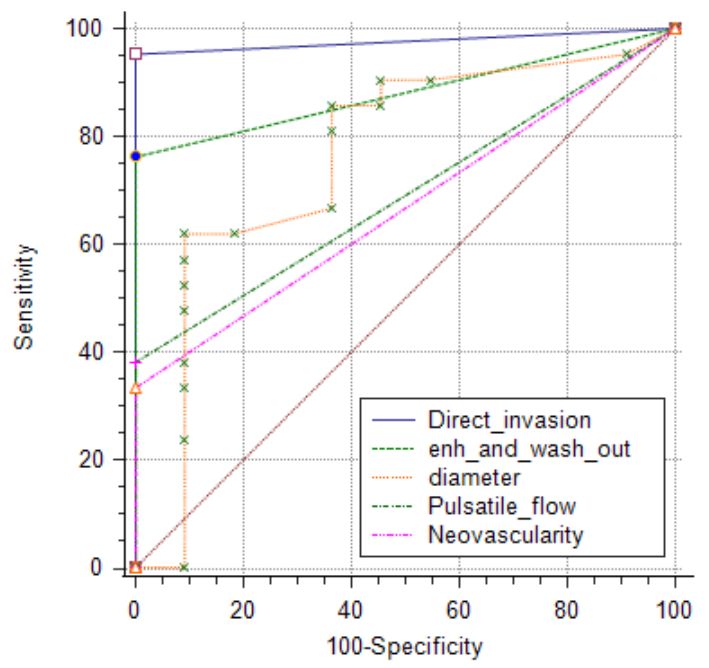

Fig.2: Receiver operating characteristic curve analyses. The specificity and sensitivity of using pulsatile flow, neovascularity, arterial enhancement with early washout, direct invasion by a hepatic mass and diameter of the thrombus in differentiation of malignant and benign PVT were compared. No significant differences were seen between the AUCs of pulsatile flow, neovascularity and diameter or between the AUCs of direct invasion and arterial enhancement or between the AUCs of arterial enhancement and diameter, yet the AUC of direct invasion was significantly greater than the AUC of diameter and the AUCs of direct invasion and arterial enhancement were significantly greater than the AUCs of intrathrombus pulsatile flow and neovascularity
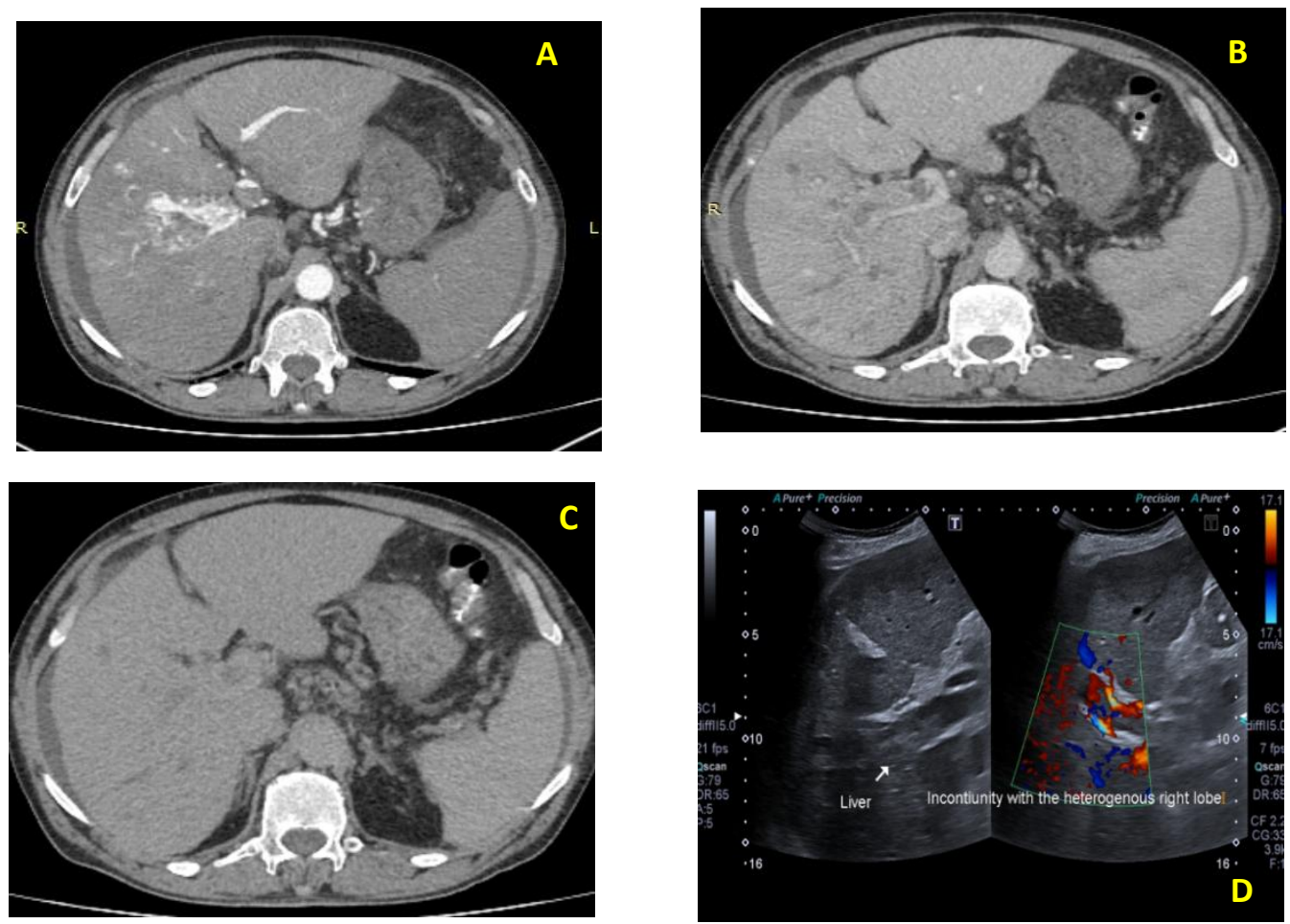

Fig. 3: A 70-year-old male patient with histopathologically proven poorly differentiated HCC. (A) Axial Triphasic CT of the liver and (B) Axial porto-venous phase revealed an enhancing thrombus and filling defect during the arterial phase with enlargment of right portal vein (arrow) shows right portal branch. (C) Axial CT delayed image shows washout of contrast. (D) Color Doppler US shows right 


\section{Eman A. Abdelsalam; et al.,}

PV thrombus and filling defect with color aliasing surrounding the thrombus due to high flow velocity (arteio-portal shunting), however no definite arterial wave could be detected within the thrombus.
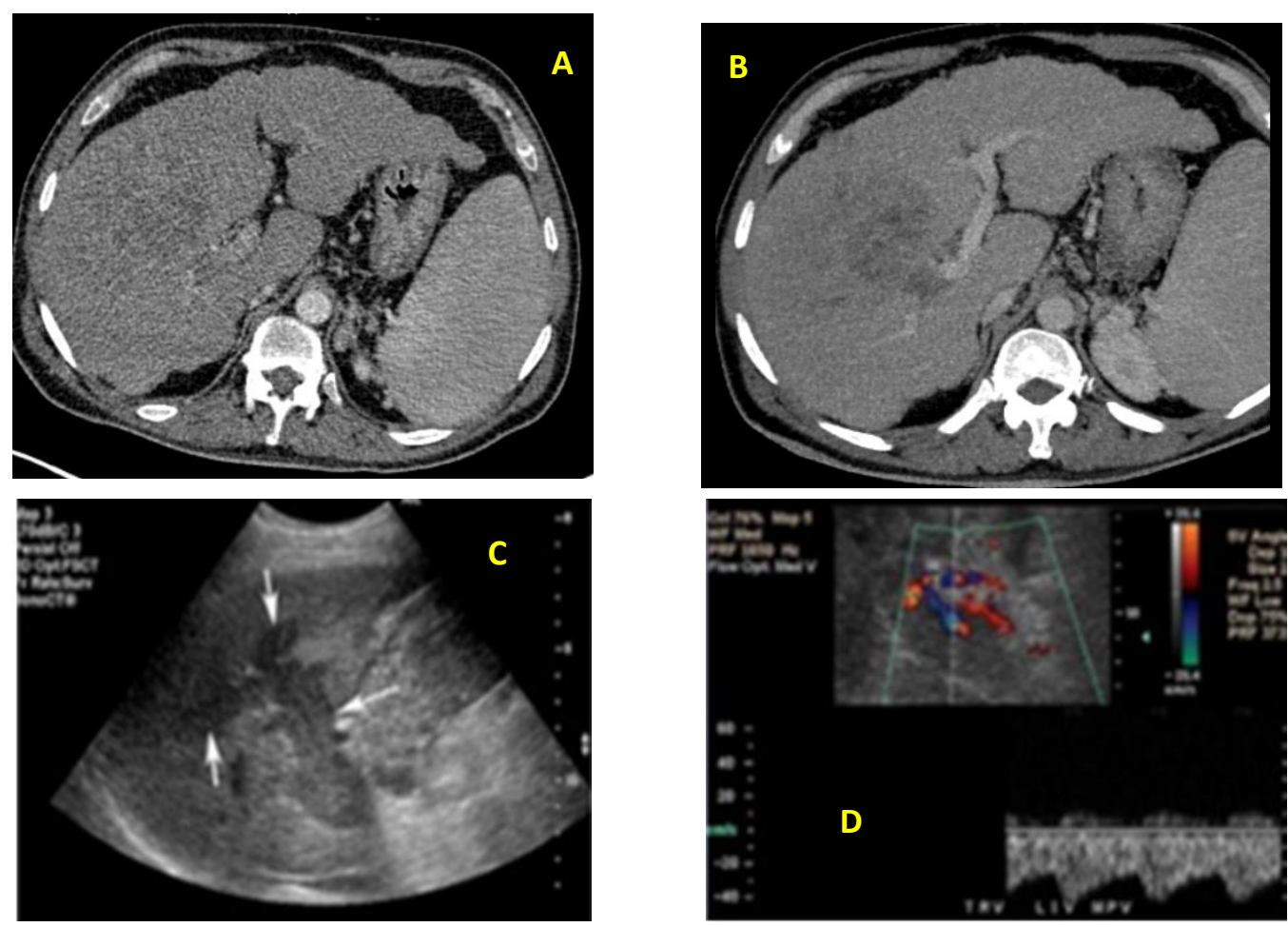

Fig. 4: A 55-year-old male patient Histopathologicaly proven as diffuse infiltrative HCC. Triphasic CT (A) Axial late arterial and (B) Axial portovenous phases shows direct invasion of PV by adjacent focal lesion with no appreciable enhancement during different phases of the study). (C) and (D) Doppler US showed arterialized wave within the right portal vein branch thrombus. Diffuse infiltrative HCC shows atypical enhancement pattern. Tumoral portal vein thrombosis suggested by direct invasion sign and Doppler examination.
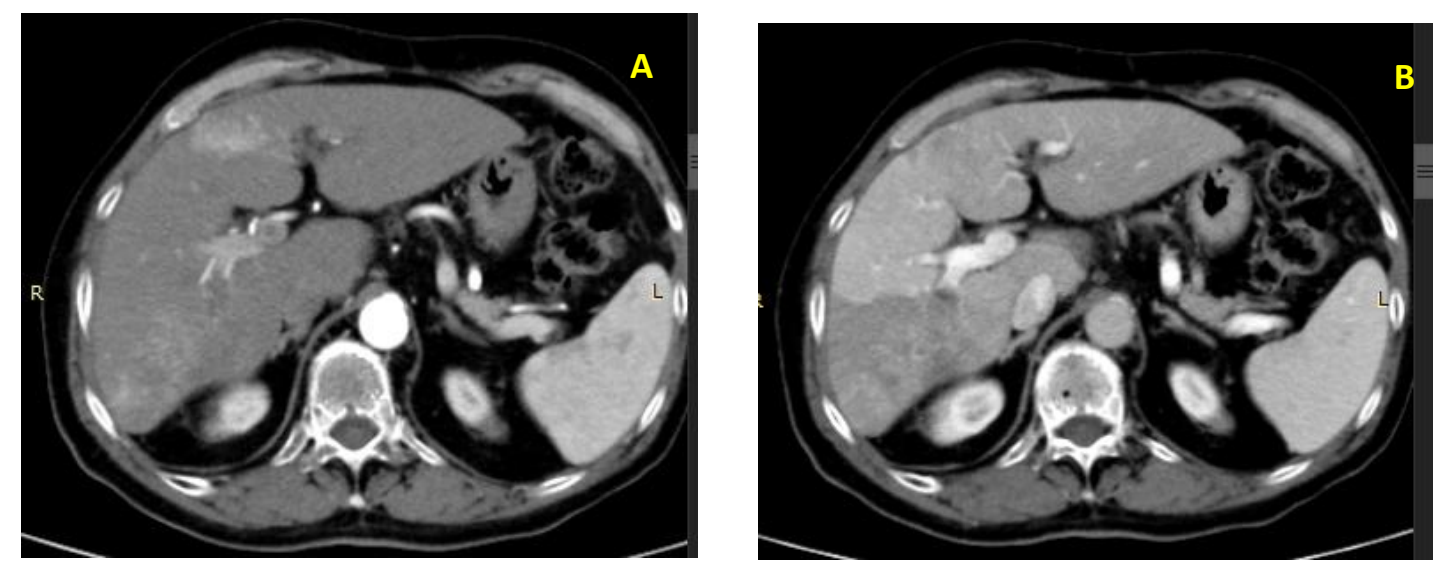

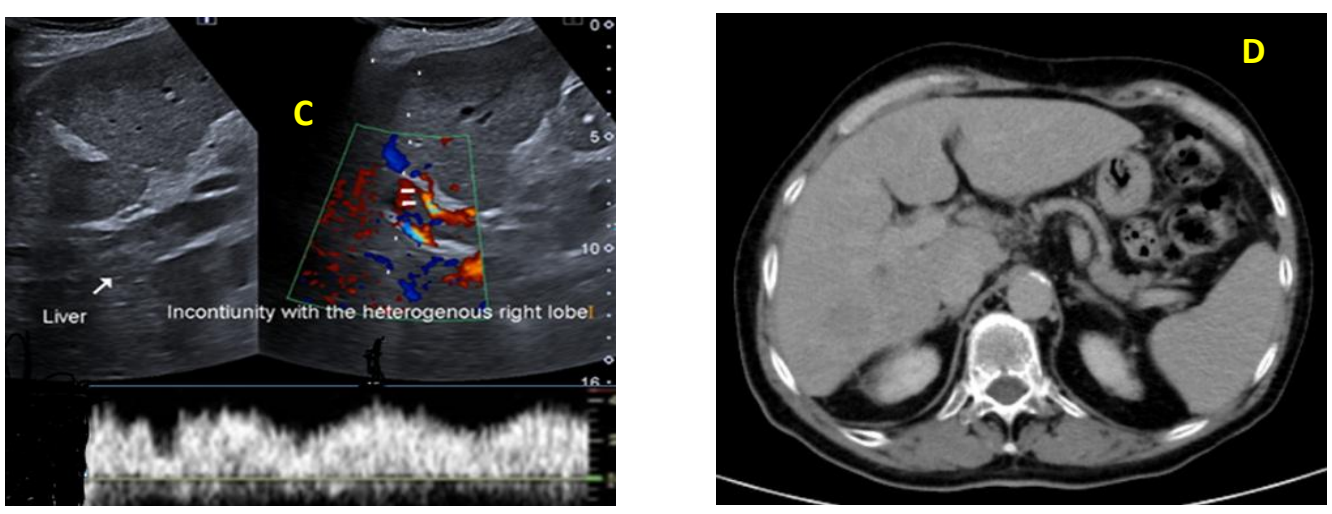

Fig. 5: A 71-year-old male patient with histopathologically proven multicentric HCC. Triphasic CT (A) Axial arterial and (B) Portovenous phases shows thrombosed right and left portal veins seen invaded by and in continuity with adjacent focal lesion with no appreciable arterial enhancement noted with both thrombi. (D) Doppler US revealed arterial wave within right portal branch thrombus.

\section{DISCUSSION:}

The discrimination of neoplastic PVT from bland thrombosis is very crucial as malignant portal vein thrombosis renders the patient unsuitable for most of the therapeutic options including surgical resection, liver transplantation, and chemoembolization, such patients usually undergo palliative treatment. On the other hand, patients with bland PVT are considered for radical treatments $^{(1)}$. In our study 25 males and 7 females with PVT are included. Liver cirrhosis is a common predisposing factor for PVT, but acquired and inherited clotting abnormality may play a role ${ }^{(1)}$. In the current study, liver cirrhosis was found in 18 patients with malignant PVT (18/21) (85\%) and in 9 patients out of 11 patients with benign PVT (9/11) (81\%). No statistical difference between patients having liver cirrhosis in malignant and benign groups, but we found high incidence of cirrhotic liver disease in cases of malignant PVT. 21 patients with malignant PVT, 18 patients of them were suffering from HCC $(85 \%)$ and 3 patients having cholangiocarcinoma (14\%). The use of Doppler sonography in the diagnosis of malignant PVT has been evaluated in many studies, absent flow within PV with pulsatile flow within the thrombus made the diagnosis. In our study 8 patients out of 21 patients with malignant PVT have intra thrombus pulsatile flow on color Doppler US, (8/21), (38\%), while the 11 patients with benign PVT had no intrathrombus pulsatile flow, with $100 \%$ specificity. This was in agreement with the results of the studies by Osman N \&Samy L $^{(2)}$, Pozniak \& Baus., ${ }^{(7)}$, Rossi et al., ${ }^{(8)}$ and Tarantino etal., ${ }^{(9)}$ who stated that all the patients with benign PVT had no intrathrombus pulsatile flow with specificity $100 \%$.

Triphasic CT is one of the best diagnostic modalities of PVT and for evaluation of the causative disease $^{(2)}$. At current study, on CT we found that out of 21 patient have malignant PVT, 7 patients (33 $\%)$ showed neovascularity of portal vein thrombus, with sensitivity of $33.3 \%$, specificity $100 \%$ and accuracy $56.3 \%$. In the study by Osman N \& Samy L., (2), they found that out of 33 patients with malignant PVT, 28 patients showed neovascularity of the thrombus with sensitivity of $84.8 \%$ and specificity of $100 \% .16$ Patients with malignant PVT pathologically proven HCC (76\%) of showed early arterial enhancement and rapid washout of the thrombus with sensitivity of $76.2 \%$, specificity $100 \%$ and 
accuracy $84.4 \%$. two cases with pathologically proven infiltrative HCC showed no appreciable arterial enhancement.

Histopatholgically diagnosed three patients with cholangiocarcinoma showed no arterial enhancement of the thrombus. Our results were in agreement with those of Osman N \&Samy L., ${ }^{(2)}$, Lee et al., ${ }^{(8)}$ who considered early arterial enhancement and rapid washout of contrast is $100 \%$ specific for malignant PVT. Also we found that out of 21 patients with malignant PVT 13 showed PVT dimensions more than $18 \mathrm{~mm}$ with sensitivity of $61.9 \%$, specificity of 90.9 $\%$ and accuracy of $71.9 \%$

On the other hand none of the 11 patients with benign PVT showed no neovasularity of the thrombus and no early arterial enhancement and rapid washout of the thrombus and the diameter of PV thrombus was less than $18 \mathrm{~mm}$.

20 patients out of 21 patients $(95.2 \%)$ with malignant PVT showed direct invasion of portal vein by adjacent hepatic focal lesion with sensitivity $95.2 \%$, specificity $100 \%$ and accuracy $96.9 \%$. Direct invasion of portal vein is more appreciated in our study in comparison to (Osman N \&Samy L., 2016) in which only $75.7 \%$ of malignant PVT showed direct PV invasion.

In our study we compared diagnostic variables through the AUCs of their ROC curves, we found that the AUC of direct invasion was greater than that of arterial enhancement and washout, no significant difference was found between their respective AUCs indicating that the diagnostic performance of these two parameters did not differ significantly. However the AUCs of direct invasion and arterial enhancement were significantly greater than the AUCs of intra-thrombus pulsatile flow and neovascularity indicating that direct invasion and arterial enhancement were better than pulsatile flow and neovascularity in differentiation of malignant and benign PVT.

No significant differences were seen between the AUCs of pulsatile flow, neovascularity and diameter or between the AUCs of arterial enhancement and diameter, yet the AUC of direct invasion was significantly greater than the AUC of diameter.

Consequently, the specificity for the diagnosis of malignant PVT approaches 100 $\%$. If there is at least two of the following findings: intra thrombus pulsatile flow, direct invasion of portal vein by adjacent focal lesion, intra thrombus neovascularity and early arterial enhancement and rapid washout of contrast.

We decided to rely on imaging criteria as the reference standard in PVT differentiation, taking into consideration the invasive nature of fine needle aspiration cytology with associated risks of bleeding.

\section{Conclusion:}

Combined assessment of portal vein thrombus using color Doppler US and Triphasic CT with portography is essential for more accurate evaluation and differentiation between the benign and malignant thrombus. Direct invasion of portal vein from adjacent focal lesion had the highest sensitivity $95.2 \%$ was achieved, with a specificity of $100 \%$. The Positive predictive value (PPV) was $100 \%$, while the negative predictive value (NPV) was $91.7 \%$. Early arterial enhancement and rapid washout of contrast showed $76.2 \%$ sensitivity and $100 \%$ specificity, PPV $100 \%$ and NPV $68.8 \%$. Pulsatile flow within the thrombus depicted $38.1 \%$ sensitivity, $100 \%$ specificity with PPV $100 \%$ and NPV $45.8 \%$. 


\section{REFERENCES:}

1. Gawande R, Jalaeian H, Niendorf E, Olgun D, Krystosek L, Rubin N, Spilseth B. MRI in differentiating malignant versus benign portal vein thrombosis in patients with hepatocellular carcinoma: Value of post contrast imaging with subtraction. European Journal of Radiology. 2019; 118:88-95.

2. Osman NM, Samy LA. Benign and malignant portal venous thrombosis: Multimodality imaging evaluation. The Egyptian Journal of Radiology and Nuclear Medicine. 2016;47(2):387-97.

3. Canellas R, Mehrkhani F, Patino M, Kambadakone A, Sahani D. Characterization of portal vein thrombosis (neoplastic versus bland) on CT images using software-based texture analysis and thrombus density (Hounsfield units). American Journal of Roentgenology. 2016; 207(5): W81-7.

4. Margini C, Berzigotti A. Portal vein thrombosis: The role of imaging in the clinical setting. Digestive and Liver Disease. 2017; 49(2):113-20.
5. Iranpour P, Lall C, Houshyar R, Helmy M, Yang A, Choi JI, Ward G, Goodwin SC. Altered Doppler flow patterns in cirrhosis patients: an overview. Ultrasonography. 2016; 35(1):3.

6. Rossi S, Ghittoni G, Ravetta V, Viera FT, Rosa L, Serassi M, et al. Contrast-enhanced ultrasonography and spiral computed tomography in the detection and characterization of portal vein thrombosis complicating hepatocellular carcinoma. Eur Radiol2008; 18:1749-56.

7. Pozniak MA, Baus KM. Hepatofugal arterial signal in the main portal vein: an indicator of intravascular tumor spread. Radiology 1991; 180:663-6.

8. Tarantino L, Francica G, Sordelli I, et al. Diagnosis of benign and malignant portal vein thrombosis in cirrhotic patients with hepatocellular carcinoma: color Doppler US, contrast-enhanced US, and fine-needle biopsy. AbdomImag 2006; 31:537-44.

9. Lee WK, Chang SD, Duddalwar VA et-al. Imaging assessment of congenital and acquired abnormalities of the portal venous system. Radiographics. 2011; 31 (4): 905 26. 


\section{دور الدوبلر الملون والاشعة المقطعية ثلاثية المراحل فى جطات الوريد البابى}

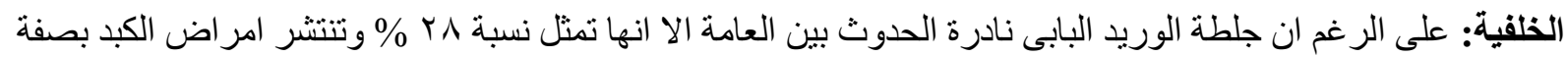

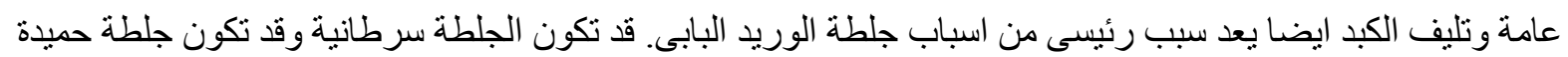

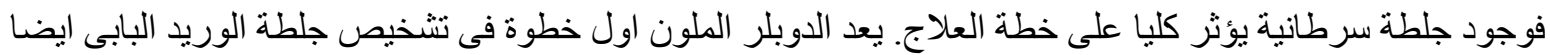

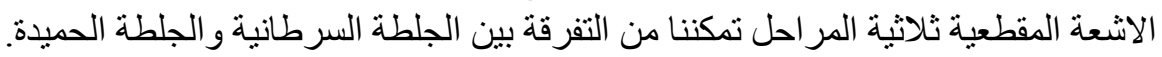

الهُف: الهدف من الرسالة ايضاح دور الدوبلر الملون والاشعة المقطعية ثلاثية المر احل فى تشخيص جلطات الوريد البابى و التفرقة بين الجلطة الحميدة و السرطانية.

المرضى والطرق: لقد قمنا بدر اسة بـ مريض يعانون من جلطات بالوريد البابى.

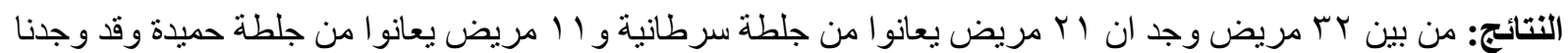

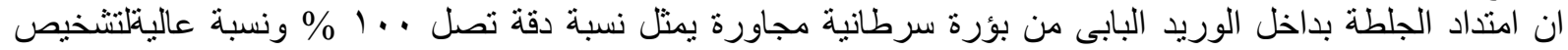

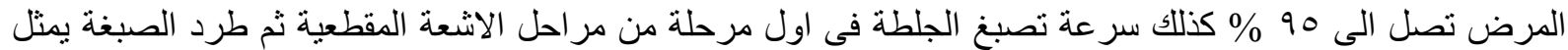

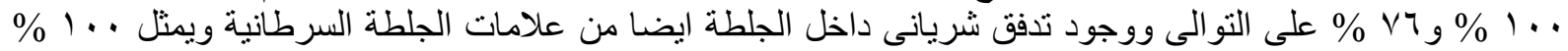

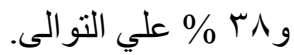

الخلاصة: الاشعة المقطعية ثلاثية الملر احل والدوبلر الملون لهم دور مهم فى توصيف جلطة الوريد البابى. 